\title{
Algorithmic trading strategy, based on GARCH $(1,1)$ volatility and volume weighted average price of asset
}

\author{
Simranjit Singh Kohli ${ }^{1}$, Nikunj Makwana ${ }^{2}$, \\ ${ }^{1}$ (Computer Engineering, Sardar Patel Institute of Technology/ Mumbai University, India) \\ ${ }^{2}$ (electronics and Communication Dept., Nirma Institute of Technology, India)
}

\begin{abstract}
Algorithmic trading strategies have one of the most significant roles for the new era of financial market. Various Hedge funds, Mutual funds and other investment banks are widely using various algorithmic trading strategies for risk management and future volatility estimation for financial instruments. In this paper, we focus upon one algorithmic approach with the aspect of special case of GARCH model, its ability to deliver volatility forecasts and moving average with multiple weighted price of asset. This model is useful not only for modeling the historical process of volatility but also in giving us multi-period ahead forecasts and helps to give exact entry price.
\end{abstract}

Keywords: Algorithmic trading, Garch,volatility, risk management,mathematical modeling

\section{Introduction}

Algorithmic trading has been one of the most prominent recent trends in financial industry. It is widely used by investment banks, mutual funds, and other buy side (investor driven) institutional traders. Sell side traders, such as market makers and somehedge funds, provide liquidity to the market, generating and executing orders automatically. This has resulted in a dramatic change of the market microstructure, particularly in the way liquidity is provided. A third of all European Union and United States stock trades in 2006 were driven by automatic programs, or algorithms, according to Boston-based financial services industry research and consulting firm Aite Group. As of 2009, HFT firms account for $73 \%$ of all US equity trading volume. This indicates the significance of robust and reliable need of trading algorithms and mathematical models for this new era of financial market to model volatility.The GARCH (p, q) model, introduced by Bollerslev (1986), often provides a parsimonious representation of the volatility dynamics in financial time series [1].

One traditional difficulty in constructing GARCH based models is that the volatility process is inherently unobservable. We surmount this problem by using a proxy of monthly volatility calculated using daily data. Moreover GARCH models treat heteroscedasticityas a variance to be modeled. As a result, not only are the deficiencies of least squares corrected, but a prediction is computed for the variance for each error term [2].We have more faith in the reliability of these volatility estimates.

\section{Need for forecasting volatility for a model}

The main purposes of forecasting volatility are measuring the potential future profit and losses of a portfolio of financial assets. Moreover it helps a lot in asset pricing phenomenon. One of the most common use of volatility for any commodity, options, stocks are to find out next day's volatility based on historical volatility. This helps to know approximate value of reruns over investment. Several recent studies have found that the volatility of daily U.S. dollar exchange rates tends to be highly persistent and well approximated by an integrated or long memory-type GARCH process[3].In asset allocation, the Markowitz approach of minimizing risk for a given level of expected returns has become a standard approach, and of course an estimate of the variance-covariance matrix is required to measure risk. Perhaps the most challenging application of volatility forecasting, Seasonality in financial-market volatility is pervasive. the historical variance of the Standard and Poor's composite stock-price index in October is almost ten times the variance for March; see also Schwert (1990) and Glosten, Jagannathan, and Runkle (1993) [4].So in today's highly volatile market,It is important to specify various parameters which help to derive volatility more accurately. High kurtosis exists within financial time series of high frequencies (observed on daily or weekly basis). This confirms the fact that distribution of returns generated by GARCH$(\mathrm{p}, \mathrm{q})$ model is always leptokurtic, even when normality assumption is introduced. Right combination of volatility parameter will help to give more reliable and accurate value of volatility. It is important to note that kurtosis is both a measure of peak and fat tails of the distribution. So we have tried to make it as accurate as possible.In the vast empirical finance literature models are well known within the GARCH framework where alternative assumptions on the conditional distribution have been suggested and extensively analyzed [5]. 


\section{Kurtosis Of Garch(1,1) Process}

GARCH models are very popular for representing the dynamic evaluation ofvolatility of financial returns.[see, e.g., Bollerslev, Engle, and Nelson (1994), Engle (1994), Bera and Higgins (1995), Diebold and Lo'pez (1995), and McAleer and Oxley (2003), among many others [6].

$\operatorname{GARCH}(1,1)$ process: has been assumed

$$
\begin{aligned}
& r_{t}=\varepsilon_{t} \\
& \varepsilon_{t}=u_{t} \sqrt{\sigma_{t}^{2}} ; u_{t} \approx \text { i.i.d.N } N(0,1), \\
& \sigma_{t}^{2}=\alpha_{0}+\alpha_{1} \varepsilon_{t-1}^{2}+\beta_{1} \sigma_{t-1}^{2}
\end{aligned}
$$

The second moment of innovation process $\left\{\varepsilon_{t}\right\}$ equals:

$$
E\left[\varepsilon_{t}^{2}\right]=\operatorname{Var}\left[\varepsilon_{t}\right]=\frac{\alpha_{0}}{1-\alpha_{1}-\beta_{1}},
$$

While the fourth moment is given as:

$$
E\left[\varepsilon_{t}^{4}\right]=\frac{3 \alpha_{0}^{2}\left(1+\alpha_{1}+\beta_{1}\right)}{\left(1-\alpha_{1}-\beta_{1}\right)\left(1-3 \alpha_{1}^{2}-2 \alpha_{1} \beta_{1}-\beta_{1}^{2}\right)^{(3)}}
$$

From covariance stationary condition of $\operatorname{GARCH}(1,1)$ process, and strictly positively conditional variance:

$$
\begin{aligned}
& 1-\alpha_{1}-\beta_{1}>0 \\
& \alpha_{0}>0
\end{aligned},
$$

Follows that the second moment of $\left\{\varepsilon_{t}\right\}$ process exists. To assure the existence of the fourth moment, apart from conditions in (4), it is necessary in relation (3) to satisfy this restriction:

$$
3 \alpha_{1}^{2}+2 \alpha_{1} \beta_{1}+\beta_{1}^{2}<1 \text {. }
$$

Since kurtosis is defined as:

$$
k=\frac{E\left[\varepsilon_{t}^{2}\right]}{\left(E\left[\varepsilon_{t}^{4}\right]\right)^{2}},
$$

then expression (6) becomes:

$$
k=\frac{3\left(1+\alpha_{1}+\beta_{1}\right)\left(1-\alpha_{1}-\beta_{1}\right)}{1-3 \alpha_{1}^{2}-2 \alpha_{1} \beta_{1}-\beta_{1}^{2}} .
$$

After some rearrangement in (7) we can write:

$$
k=3+\frac{6 \alpha_{1}^{2}}{1-3 \alpha_{1}^{2}-2 \alpha_{1} \beta_{1}-\beta_{1}^{2}} .
$$

From relation (8) follows that distribution of returns generated from $\operatorname{GARCH}(1,1)$ process always results in excess kurtosis, i.e. Fisher's kurtosis $(k>3)$ even normality assumption is introduced, if and only if conditions in (4) are satisfied. These conditions also could be satisfied when parameter $\alpha_{1}=0$. Only in that case innovations distribution would be normally shaped ( $k=3$ ). Therefore, the kurtosis is very sensitive on value of parameter $\alpha_{1}$.In general kurtosis increases much intensively with larger parameter $\alpha_{1}$ in comparison to parameter $\beta_{1}$.

\section{Degrees Of Freedom Estimation}

Generally, there are three parameters that define a probability density function: (a) location parameter, (b) scale parameter and (c) shape parameter. The most common measure of location parameter is the mean. The scale parameter measure variability of probability density function $(p d f)$, and the most commonly used is variance or standard deviation. The shape parameter (kurtosis and/or skewness) determines how the variations are distributed about the location parameter.

If the data are heavy tailed, the VaR calculated using normal assumption differs significantly from Students t-distribution. Therefore, we find that kurtosis and degrees of freedom from Student's distribution are closely related. 
Algorithmic trading strategy, based on $\operatorname{GARCH}(1,1)$ volatility and volume weighted average price of The density function of no central Student t-distribution is given as:

$$
f(x)=\frac{\Gamma\left(\frac{d f+1}{2}\right)}{\Gamma\left(\frac{d f}{2}\right) \sqrt{\pi \cdot \beta \cdot d f}}\left(1+\frac{(x-\mu)}{\beta \cdot d f}\right)^{-\frac{1+d f}{2}}
$$

Where $\mu$ is location parameter, $\beta$ scale parameter and $d f$ shape parameter, i.e. degrees of freedom, and $\Gamma($.$) is gamma function. Standard Student's t-distribution assumes that \mu=0, \beta=1$, with integer degrees of freedom. However, degrees of freedom can be estimated as non-integer, relating to kurtosis:

$$
k=\frac{6}{d f-4}+3 \quad \forall d f>4 .
$$

From relation (10) it's obvious that standard t-distribution has heavier tails than normal distribution when $4<d f \leq 30$. Hence, if empirical distribution is more leptokurtic estimated degrees of freedom would be smaller.

The second and fourth central moment of function (9) are defined as:

with Fisher's kurtosis:

$$
\begin{aligned}
& \mu_{2}=E\left[(x-\mu)^{2}\right]=\frac{\beta \cdot d f}{d f-2} \\
& \mu_{4}=E\left[(x-\mu)^{4}\right]=\frac{3 \beta^{2} d f^{2}}{(d f-2)(d f-4)},
\end{aligned}
$$

$k^{*}=\frac{\mu_{4}}{\mu_{2}^{2}}-3=\frac{6}{d f-4}$.

Therefore, we may apply method of moments and get consistent estimators:

$d \hat{f}=4+\frac{6}{\hat{k}^{*}}$

$\hat{\beta}=\left(\frac{3+\hat{k}^{*}}{3+2 \hat{k}^{*}}\right) \hat{\sigma}^{2}$

Where the sample variance is biased estimator of $\beta$.To get unbiased estimator of standard deviation we use correction factor:

$\sqrt{\frac{3+\hat{k}^{*}}{3+2 \hat{k}^{*}}}$

which is equivalent to:

$\sqrt{\frac{d \hat{f}-2}{d \hat{f}}}$.

In practice, the kurtosis is often larger than six, leading to estimation of non-integer degrees of freedom between four and five. However, kurtosis will depend on volatility persistence. Volatility persistence is defined as the sum of parameters $\alpha_{1}+\beta_{1}$ in $\operatorname{GARCH}(1,1)$ model.

If we rearrange condition variance equation of $\operatorname{GARCH}(1,1)$ model as follows:

$\sigma_{t}^{2}=\alpha_{0}+\alpha_{1}\left(\varepsilon_{t-1}^{2}-\sigma_{t-1}^{2}\right)+\left(\alpha_{1}+\beta_{1}\right) \sigma_{t-1}^{2}$,

Then the sum of parameters $\alpha_{1}+\beta_{1}$ shows the time which is needed for shocks in volatility to die out. If this sum is close to 1 long time is needed for shocks to die out. However, if the sum is equal to unity the covariance stationary condition is not satisfied and GARCH$(1,1)$ model follows integrated GARCH process of order one, i.e. $\operatorname{IGARCH}(1,1)$.

If we substitute $\sigma_{t}^{2}=\varepsilon_{t}^{2}-v_{t}$ than stationary condition occurs from $\operatorname{ARMA}(1,1)$ representation of $\operatorname{GARCH}(1,1)$ model:

$\varepsilon_{t}^{2}=\alpha_{0}+\left(\alpha_{1}+\beta_{1}\right) \varepsilon_{t-1}^{2}+v_{t}-\beta_{1} v_{t-1}$. 


\subsection{Volatility Calculation}

\section{Proposed Algorithm}

Volatility parameters of $\operatorname{GARCH}(1,1)$ like variance - covariance matrix, Kurtosis, probability density function are calculated on basis of historical data. We have shown empirical results for last 14 years for S\&P 500 in Table 1.

\subsection{Boundary value calculation}

Once volatility parameters are calculated, they are fed to the equation from which boundary value is obtained. Boundary values are weighted average price of the financial Instrument over multiple time periodsas Volume Weighted Average Price (VWAP) of an asset is a well-established benchmark [7]. This boundary value acts as the reference for the decision support system.

\subsection{Decision Support System}

Economic theory frequently suggests that economic agents respond not only to the mean, but also to higher moments of economic random variable [8].

On obtaining the boundary value the order is executed by the decision making system. If the opening value is greater than boundary value the conclusion is reached that the instrument is overvalued. Hence short position is taken with selling point being the difference between opening value and predicted volatility. On the other hand if the opening value is less than the boundary value the conclusion is reached that the instrument is undervalued. Hence, long position is taken with the selling point being the sum of volatility and opening. It can be noted that for both cases the profit is volatility.

\subsection{Risk Management Process for the algorithm}

The risk management process must also take place in real time, alongside with the algorithm parameter outputs and order management. The value of the open positions and cash available must be carefully correlated with the terms settled when the algorithm starts during any sort of security trading stop loss is necessary. In the risk management module the current prices are monitored constantly and checked against a stop loss value. See figure 2 for Risk Management Process.

One important issue is the one of tuning the parameters of algorithm. Even if in practice there are some standard values for them, the performance of the algorithm can be much affected by a non-optimum parameter value chosen. The main factors that lead to parameters changing are the volatility estimation parameters.

Fuzzy logicor neural network algorithms can also be used to overcome the issue of choosing right parameters among multiple combination sets. The risk management process is responsible not only for the loss limitation but also for cashing the profit. So it has immense significance for real-time environment.

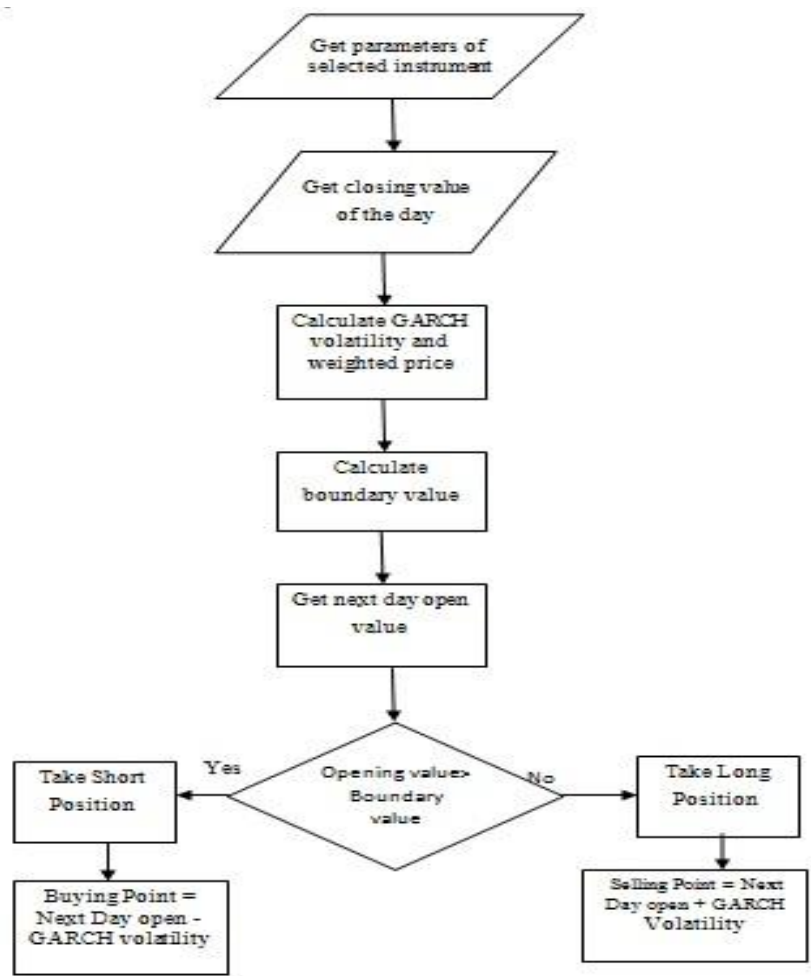

Figure 1. Algorithm Flowchart 


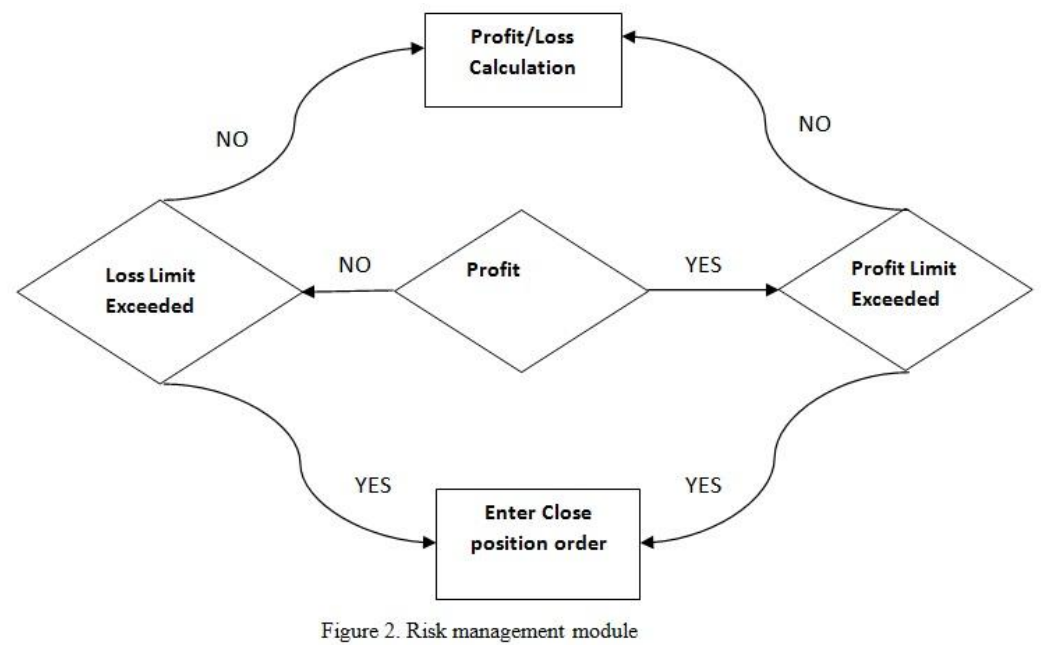

VI. Empirical Results:

The findings with the algorithms are presented here. The performance of the S\&P 500 has been analyzed. Monte-Carlo simulation has been performed over random subset of stocks. We use daily data to make forecasts for the next day and have overnight time interval from the close of trading open of the next day, riskfree rates are used. The transaction cost incurred is $1 \%$ when we change our position. It will be based on tomorrow's closing price because we focus on out-of-sample prediction and we assume that we place our order to buy or sell immediately before the close of trading tomorrow. Of course we may use tomorrow's opening price or high frequency data in practice. We believe that here the strategy will be more profitable because of more flexibility and less delay.

The leverage feedback effect has magnified the fluctuation in the market caused by the extreme events. For example, after Lehman Brothers declared its bankruptcy on September $14^{\text {th }}, 2008$, a series of bank and insurance company failures triggered the global financial crisis in which the market fluctuates dramatically. It is the extreme event, i.e., the declaration of Lehman Brothers' bankruptcy, together with the volatility clustering plus the leverage feedback effect caused by Lehman Brothers bankruptcy news result in the catastrophic financial crisis in 2008 [9].So leverage and transaction cost cannot be neglected. Our empirical results take both of these parameters in account. Figure 3 shows Cumulative classic return obtained via logarithmic return for S\&P 500 from 1994 to 2011.Table 1 shows all evaluation parameters of the test in detail.

Figure 3: Cumulative classic return obtained via logarithmic return for S\&P 500 from 1994 to 2011

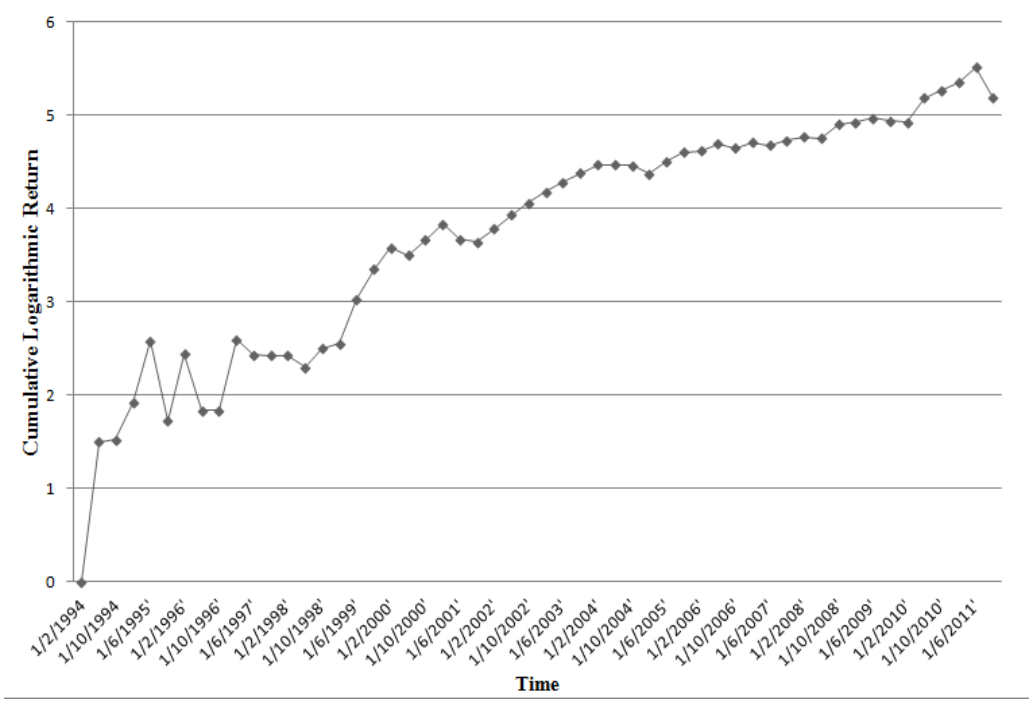




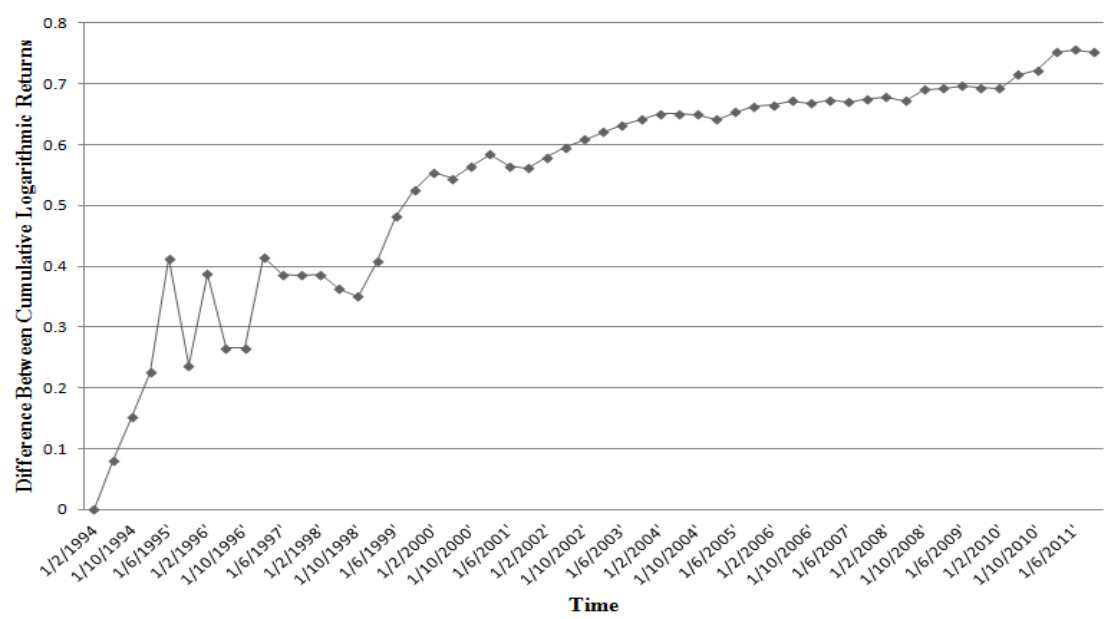

Figure 4: Time series of difference between cumulative logarithmic returns of the strategy and the proxy portfolio with S\&P 500 from 1994 to 2011

Table 1: Performance of the strategy in terms of daily logarithmic returns for S\&P 500

\begin{tabular}{|l|c|}
\hline \multicolumn{1}{|c||}{ Parameters } & Value \\
\hline \hline Logarithmic Return & $195 \%$ \\
\hline Classic Return & $580 \%$ \\
\hline Mean Daily Logarithmic return & $0.08 \%$ \\
\hline Standard Deviation & 0.0075 \\
\hline Skewness & 0.36 \\
\hline Kurtosis & 10.085 \\
\hline
\end{tabular}

\section{Conclusion}

Andersen and Bollerslev (1998) and Christodoulakis and Satchell $(1998,2003)$ have argued that the poor forecasting from GARCH models are too smooth to capture the entire variation of volatility. So we have moved one step forward. We have introduced the concept of weighted value of asset with multiple period based boundary condition along with volatility.

We have focused on one aspect of GARCH models and their ability to deliver one period ahead forecasts of volatility. We have analyzed these forecasts to actual volatility calculated using daily stock returns. Weighted price and boundary value gives us exact entry point. In the further work the integration with a Genetic Algorithm or neural network with regression analysis can be a highly desirable solution in order to tune up the parameters of the algorithm in a quick and reliable way, but also can be used in the discovery of new trading rules in a quick and reliable way.

\section{References}

[1] Bollerslev Tim Generalized Autoregressive conditional heteroscedasticity, Journal of Econometrics 31(3), $1986,307-327$.

[2] Robert F. Engle, The use of ARCH/GARCH Models in Applied Econometrics, Journal of Economic Perspectives 15(4), 2001, 157168 .

[3] Engle Robert Autoregressive Conditional Heteroscedasticity with Estimates of the Variance of United Kingdom Inflation, Econometrica 50(4), 1982, 987-1007.

[4] Glosten L Jagannathan R and Runkle D, On the Relation between the Expected Value and the Volatility of the Nominal Excess Return on Stocks Journal of Finance 48(5) , 1993 , 1779-1801.

[5] Stentoft L American Option Pricing Using GARCH Models and the Normal Inverse Gaussian Distribution ,Journal of Financial Econometrics 6(4), 2008,540-582.

[6] Carnero M, A Penam, D Riuz E, Persistence and Kurtosis in GARCH and Stohastic Volatility Models, Journal of Financial Econometrics Oxford University Press, 2 (2), 2004, 319-372.

[7] Madhavan, A. VWAP Strategies, Institutional Investor Guides: Trading Institutional Investor Inc., 2002, 32-29.

[8] Hall P Yao Q Inference in ARCH and GARCH Models with Heavy-Tailed Errors, Econometrica 71(1), $2003,285-317$.

[9] Lie-Jane Kao, Po-Cheng Wu and Cheng-Few Lee, A Time-changed NGARCH model on the leverage and volatility clustering effects by extreme events: Evidence from the S\&P 500 index over the 2008 financial crisis, Proc 3rd Annual Financial Engineering, and Risk Management Conference National Chiao Tung University 2010. 